\title{
Biomolecular Nonlinear Dynamic Mechanisms as a foundation for Human Traits of Information Processing Machine
}

\author{
NICHOLAS G. RAMBIDI \\ Department of Physics, Moscow State University, International Research Institute for Management Sciences, 2-ya, Pestchanaya 8-53, \\ Moscow, Russia
}

(Revised 3 January 2001)

\begin{abstract}
A pseudo-biological paradigm in information processing launched by McCulloch and Pitts in the early 1940s has been advanced during the last decades. Different attempts were made based on these developments to design operational information processing devices capable of solving problems of high computational complexity.

One of them was the use of nonlinear dynamic mechanisms inherent in information processing by biochemical, biomolecular, and simple biological entities. Chemical reactiondiffusion media proved to be effective tools for the implementation of these capabilities.

Basic features of these information processing means and modeling of their information processing capabilities are discussed in this paper. Belousov-Zhabotinsky type reactiondiffusion media were used to simulate image processing operations and finding paths in a labyrinth.
\end{abstract}

Keywords: Nonlinear reaction-diffusion systems; Nonlinear structures and dynamics; Solving problems of high computational complexity; Image processing; Finding paths in a labyrinth

\section{SOME PRELIMINARY REMARKS}

Could an information processing device based on absolutely different from von Neumann principles be more effective than contemporary computers that dramatically have been changing our life?

Not at the expense of sophisticated software but due to fundamentally different structural and dynamic organization?
There are several fundamental points that should be discussed to answer these questions. The most urgent among them are:

- high computational complexity of problems that are of vital importance for the progress of the human society;

- nonlinear dynamic mechanisms inherent in systems that display high behavioral complexity and 
are capable of solving problems of high computational complexity;

- the capability of reaction-diffusion systems to implement information processing operations of high computational complexity.

This paper was designed as an attempt to put these points together and to discuss potentialities to elaborate nondiscrete information processing means based on chemical reaction-diffusion systems.

\section{SEVERAL BASIC POINTS OF DEPARTURE}

The world around us is complex. And it is the reality that has been defining our understanding of different phenomena we face everyday. They reveal in the absolutely diverse fields of human activity beginning from complicated engineering designs up to sophisticated economic and social problems.

There is no need to discuss here in the problem oriented paper details of different faces of this multivalued concept (see, for instance, Beer, 1970). Let us make clear only understanding basic notions that will be used below.

Virtually these notions were formulated as far as late seventies. According to Casti (1979) complexity concept embraces three basic aspects.

"Static complexity represents essentially the complexity of the subsystems that realize the system, dynamic complexity involves the computational length required by the subsystems interconnection to realize the process, and control complexity represents a measure of computational requirements needed to keep the system behaving in a prescribed fashion."

Problems that would be discussed in the paper have a biological background. There are a lot of systems inbetween of chemistry and biology having similar behavioral characteristics. They include some tissues of living organisms, assemblies of simple microorganisms, biological membranes, sets of coupled biochemical and chemical reactions with nonlinear kinetics and so on. The term "biomolecular system" will be used below for all this entities.
Following Casti (1979) let us consider three basic hypostases of the complexity inherent in biomolecular systems capable of information processing. Given the biological background of the problems discussed let us use terms:

- structural complexity of the system (static);

- behavioral complexity that determined the spatiotemporal evolution of the system performing an information processing operation (dynamic);

- computational complexity of an algorithm describing information processing operations performed (control).

Several approaches to the quantitative definition of complexity are in use now. The notion of algorithmic complexity seems to be the most adequate to the complexity estimation of the dynamic system evolution (Yudin and Yudin, 1985; Ming and Vitanyi, 1993). It is defined as minimal length of the assumed program from some set of programs that describe the process adequately.

Numerical estimations of algorithmic behavioral and structural complexity are not used in the paper. All examples that will be discussed below are chosen in a way when the high (or low) degree of behavioral complexity is evident.

Computational complexity of the algorithm describing the system behavior (complexity of the problem) is a characteristic of the practical opportunities to understand this behavior. It can be expressed as a dependence of computational capabilities (resources) necessary for simulation of the system behavior on some specific system characteristic called the problem size (Casti, 1979; Yudin and Yudin, 1985; Ming and Vitanyi, 1993).

Two basic principles could be set based on experimental experience and theoretical considerations that determine high behavioral complexity of the system. They are:

- nonlinear mechanisms of the system dynamics;

- multilevel structural organization with interactions between levels.

Reaction-diffusion media such as nonlinear biochemical enzymatic systems and chemical 
oscillators represent striking examples of very complex behavior based on primitive structure.

This paper was designed:

- to give examples and to discuss high behavioral complexity of biomolecular systems based on nonlinear dynamic mechanisms;

- to speculate on the assumption that high behavioral complexity of biomolecular media is in close correlation with their capabilities to solve problems of high computational complexity;

- to give examples of complex computations performed by biomolecular media.

\section{NONLINEARITY AND BEHAVIORAL COMPLEXITY OF BIOMOLECULAR SYSTEMS}

Divers and important examples of high behavioral complexity were shown by biomolecular and biological systems at different levels of organization (structure).

At the tissue level complex oscillatory processes in a human brain are known. Heart rhythm disturbances and sudden death phenomenon are determined by pathological modes of myocardium excitations (Winfree, 1994).

At the level of sell assemblies complex dynamic regimes lead to the ordered spatial evolution, for instance, formation of nonuniform circular cell distributions in thin layers of DICTYOSTELLUM DISCOIDEUM media (Prigogine, 1980).

Complex concentration oscillating modes were found for divers chemical and biochemical reactions in biological membranes and cells, that is at supramolecular level (Goldbetter, 1997).

And finally in biomacromolecules, at the molecular level, complex dynamics could be the origin of collective excitations (Davydov, 1984).

One of the basic points for understanding features of information processing system functioning is the correlation between its structural and behavioral complexity. It is quite often assumed, for instance for the case of electronic technical systems, that behavioral complexity should increase proportionally to increasing structural complexity. Nevertheless, the correlation between structural and behavioral complexity is not so straightforward. The complications of the system structure do lead in many cases to the more complicated behavior. At the same time some very simple systems (for instance, two oscillators with nonlinear coupling) are known that demonstrate complex behavior in spite of the simplicity of their structure (Nicolis, 1986).

Chemical reaction-diffusion systems (for instance, Belousov-Zhabotinsky media) demonstrate different complex modes of behavior such as concentration oscillations, waves of switching between different states, traveling concentration pulses, stable dissipate structures and so on (Field and Burger, 1985). Two examples of temporal evolution of the BelousovZhabotinsky system are shown in Fig. 1.

It is known (Field and Burger, 1985) that the dynamics of distributed nonlinear chemical system which display sufficiently complicated behavior can be described by a system of nonlinear differential equations of the type:

$$
\begin{aligned}
U_{i}(r, t)= & F\left[U_{1}(r, t), U_{2}(r, t), \ldots, U_{N}(r, t)\right] \\
& +\sum_{j=1}^{N} \Delta\left[D_{i j} U_{j}(r t)\right]
\end{aligned}
$$

where $U_{i}(r, t)$ is the concentration of the $i$ th component of reaction proceeding in the system, $A$ a control parameter, $D_{i j}$ the diffusion coefficients, and

$$
N=1,2,3, \ldots, N \text {. }
$$

The behavior of this system is determined by the complicated nonlinear kinetics of reactions at each spatial point $r_{k}$, described by functions

$$
F\left[U_{1}(r, t), U_{2}(r, t), \ldots, U_{N}(r, t)\right]
$$

and also by diffusion of reaction components.

At the same time such an excitable system can be considered as a realization of a neural network where:

- each point of the medium is a primitive microprocessor; 
- the dynamics of microprocessors can be characterized by complicated chemical reactions produced by external excitations;

- short-range interactions between primitive processors occur (in principle, each microvolume is coupled with all others by diffusion, but because of a rather low spreading speed, these interactions proceed with a delay proportional to the distance between microvolumes).

In the general form homogeneous neural nets can be described by a system of integro-differential equations (Masterov et al., 1988):

$$
\begin{aligned}
U_{i}(r, t)= & -\frac{U_{i}(r, t)}{t_{i}}+G\left[-T_{i}-A+Z_{i}\right] \\
& =-\frac{U_{i}(r, t)}{t_{i}}+G\left\{-T_{i}-A+\sum_{m=1}^{N} \int \Phi_{m}\right. \\
& \left.\times\left[r, x, t, U_{1}, U_{2}, \ldots, U_{N}\right] U_{m}(r, t) \mathrm{d} x\right\}, \quad(2)
\end{aligned}
$$

where $G\left[-T_{i}-A+Z_{i}\right]$ is the response function for elements of $i$ th type upon activation by $Z_{i}, T_{i}$ is the shift in function $G, \Phi_{m}$ is the function of spatial coupling between active elements.

These integro-differential equations cannot generally be represented by the above system of kinetic differential equations (see Vasilev et al., 1987). However, under some assumptions both of these models prove to be adequate.

The remarkable feature of biomolecular systems based on nonlinear dynamic mechanisms is that they are capable to fulfill functions adequate to information processing operations of high computational complexity. Besides fantastic intellectual capabilities of a neural system let us mention processes of information replication performed by RNA molecules, recognition at the molecular level inherent in enzyme molecules and so on.

This feature of biomolecular systems is in the basis of pseudo-biological information processing paradigm that is an important alternative to the unique von Neumann approach in the contemporary digital computing.

\section{PSEUDO-BIOLOGICAL VS. VON NEUMANN INFORMATION PROCESSING PARADIGMS}

Two basic methodological approaches have been defining general ways of developing contemporary computing, i.e. theoretical fundamentals, circuitry approaches, technological incarnations, and software elaboration.

General principles lying in the basis of contemporary computer designs were formulated in the early 1940s by John von Neumann. These principles makes the von Neumann approach indispensable for the elaboration of multipurpose computing systems that are capable of optimum solving engineering and many other problems of relatively low computational complexity.

Regretfully the multipurpose character of the von Neumann computers leads to the decrease of computational efficiency, especially for problems of high computational complexity.

It should be mentioned that more and more the progress in important fields of human activity is based on the ability to efficiently solve problems of high computational complexity. Among them are:

- recognition of images, scenes, and situations that can be conditionally divided into a number of steps, such as:

- classification of the object according to a definite set of features;

- segmentation of the object into the simplest (for this object) fragments;

- recognition of the fragments in context, designing the image based on these fragments;

- investigation of the evolution of systems having complicated behavioral dynamics (for instance, tasks of "predator-victim" type, dynamics of cell populations, etc.);

- choice of optimal (in some predetermined sense) structure of a multifactor system having complicated branching search trees (combinatorial problems);

- control problems that embrace: 
- continuous recognition of situations based on associative bounds;

- continuous in general case choice of optimum strategy (decision making).

At the same time, when von Neumann had elaborated his paradigm, McCulloch and Pitts (1943) offered fundamentally different approach to designing information processing devices. This approach was based on general principles of information processing by biological entities and was called pseudo-biological one.

According to ideas of McCulloch and Pitts (1943) computational system is designed to be analogous in a sense to human brain. Simple processors (neurons) are constituent parts of the system and each of them is connected to all other processors in some definite manner. Therefore, computing capabilities of the system are defined by the predetermined complex structure of the system, not by the stored program. Problems are solved by the system with very high degree of parallelism. At the same time storage of information and information processing capabilities of the system are defined by the character of dynamics inherent in the system.

The relative significance of the von Neumann and McCulloch and Pitts paradigms was different during the last decades depending on the character of those practically important problems that were the most vital at that moment for the progress of human activity.

Virtually the computational complexity of problems was of the decisive importance for the choice of either paradigm.

The vast variety of divers engineering projectskeystones for industry, developing in the 1940s and 1950s, initiated the impetuous progress of the digital von Neumann computing means. Mathematical and computational basis for these projects could be reduced to the problems of rather low (polynomial) computational complexity.

Little by little, needs to understand to control processes in biology, meteorology, economics, social sciences and a number of other fields that was defined by problems of high computational complexity gave rise to attempts to elaborate fundamentally different from von Neumann computational approaches.

The pseudo-biological paradigm based on $\mathrm{McCul}-$ loch and Pitts pioneering work was one of the first in the line.

Neural net approach launched by McCulloch and Pitts was based on two fundamental principles inherent in information processing by biological entities. They are:

- "all-or-none" mode of a single neuron activity, that is a kind of nonlinear dynamic mechanisms;

- giant parallelism of neural connections in a neural net.

Designers of computing means have been repeatedly facing this paradigm during the last several decades. Nonetheless, only nowadays, when problems of high computational complexity do define a number of important aspects of human activity, neural net approaches began to give the practical tangible results at both software and hardware levels (see, for instance, Lee, 1989; Niels and Hepner, 1990; Trouder and Meril, 1990).

To make clear the contemporary situation, let us return to the roots, that is to the general fundamentals of biological information processing (Rambidi, 1997).

The hereditary information code unique in its designing together with specific information transfer mechanisms provides biological systems with stability in the process of reproduction. At the same time, the possibilities to modify this information in the process of evolution supplies a biological system with the ability to adapt according to the changing external stimuli.

The molecular recognition processes ensures the directed information transfer in a biomolecular system and therefore excludes the random search of variants.

The giant parallelism exceeding immensely possible degree of parallelism of contemporary digital semiconductor devices is inherent in biological information processing systems.

Important is the ability to perform as primitive complex logical operations equivalent to a big number of binary ones. 
Nonlinear mechanisms of information processing are responsible for a number of complex responses biomolecular and simple biological system to external stimuli equivalent to solving problems of high computational complexity.

Finally the multilevel architecture enables biomolecular and simple biological systems to perform information processing operations of high computational complexity.

These fundamentals are of different importance for solving problems of high computational complexity.

The nonlinear mechanisms inherent in the dynamics of distributed biomolecular systems seem to be the basic fundamental that determined the information processing for problems of high computational complexity, see details in Grossberg (1976; 1988) and Rambidi and Maximychev (1997a).

The second in the line fundamental that gives the system the ability to solve computationally complex problems is its multilevel organization. The main principles and details of organization were discussed in details by Nicolis (1986).

From these considerations and bearing in mind the speculations of the previous section, note that both behavioral complexity of the system and its ability to solve problems of high computational complexity are determined by the same fundamentals of a reactiondiffusion system. Therefore the degree of behavioral complexity could be a decisive point for the choice of a system capable to solve computationally complex problems.

Based on these considerations it is natural to broaden the boundaries of the pseudo-biological paradigm in comparison with McCulloch and Pitts original approach and particularly:

- to include in the scope of the approach distributed information processing media;

- to use biomolecular information processing systems having more complicated nonlinear dynamics than in the case of McCulloch and Pitts neural networks;

- to look for possibilities of the system multilevel organization.

\section{MIRACULOUS BELOUSOV- ZHABOTINSKY TYPE MEDIA: IMAGE PROCESSING CAPABILITIES}

Two remarkable events that happened in the early 1950s were the starting points for intense investigations in the new thrilling field between physics, chemistry and biology, where systems far from equilibrium demonstrate different and complicated modes of behavior.

Chronologically they were:

- the discovery of periodic regimes in catalytic reaction of the citric acid oxidation by Belousov (Field and Burger, 1985; Kapral and Showalter, 1995);

- the paper "The Chemical Basis of Morphogenesis" (Turing, 1952) published by Alan Turing who first discussed the problem of self organization in far from equilibrium systems.

Later, Zhabotinsky (1964) performed extensive study of Belousov reaction and developed its very conveniently modified version.

These events have initiated intense research activity on complex spatio-temporal behavior in physical, chemical and biological systems during the last several decades and provided the basis for modern theories of biological patterns and forms.

Between different chemical oscillators BelousovZhabotinsky type media plays the principal role. The nonlinear dynamics of these media are complex enough to demonstrate divers and complicated behavior (see two important examples in Fig. 1). Therefore they have become the invaluable model systems for excitable media providing deep insights into the properties of nonlinear dynamic chemical and biological systems.

The Belousov-Zhabotinsky type reaction is a catalytic oxidation of some organic substance (mainly malonic acid) by potassium bromate or some other oxidizing agent. The mechanism of this process is complex and is determined by nonlinear kinetics of intermediate stages of the process and diffusion.

At the same time these media are stable, nonhostile reagents. Furthermore the temperature range and 

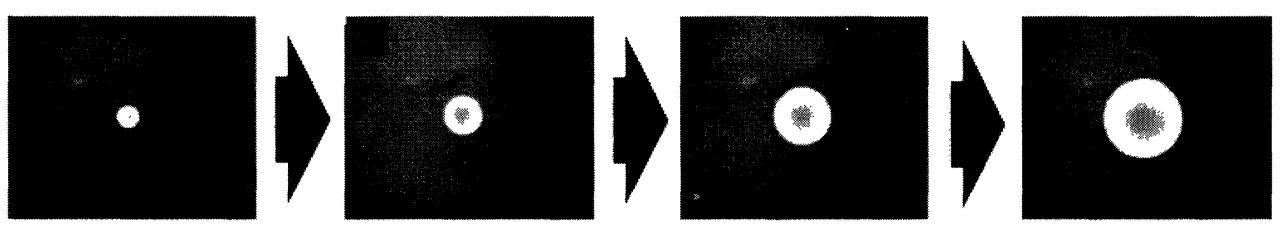

\section{A}
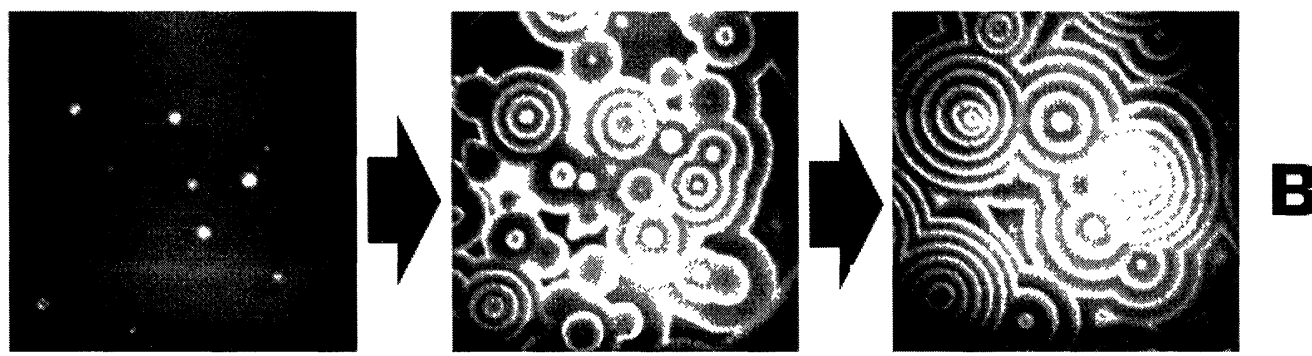

FIGURE 1 Two modes of a Belousov-Zhabotinsky reaction in thin (pseudo-two-dimensional) layers of the reagent: (A) the process of trigger wave spreading corresponding to the switching of the medium from one stable state to another; (B) the emission of circular waves from point-wise sources and their further evolution. Here and in the following figures, gray arrows show steps of the image transformation by the reaction-diffusion medium, black arrows correspond to input of an initial image into medium.

temporal operation scale of the medium dynamics are convenient for investigation with the available physical methods.

The Belousov-Zhabotinsky type media based on a light-sensitive catalyst are convenient for investigation purposes. The catalyst in the course of reaction, when the medium goes from one stable state into another, changes its electronic state. As a consequence the reagent changes its color (from red to blue and vice versa). Therefore it is easy to visualize the process and to observe its spatio-temporal evolution.

Practical experimental aspects of information processing by reaction-diffusion media are not considered in the paper. Enough of this detailed information could be found in Rambidi et al. (1994).

There is no problem to reproduce some results that will be discussed below using very simple experimental technique. Nevertheless, the complication of problems to be solved compels to design more and more sophisticated devices. The most important and decisive point is designing reaction-diffusion media (see Rambidi et al., 1998).

The key important feature of light-sensitive excitable media is that they store input information during rather long period of time. Periodical process of stored image transformation begins after projecting an image onto a thin layer of the medium (Rambidi and Maximychev, 1995).

This process represents a combination of three interlaced primitive responses to the light excitation:

- consecutive emergence of negative and positive images of an input picture;

- contour enhancement of the image fragments;

- disappearance of small features of the picture.

Revealing these primitive responses depending on the state of the medium is shown in Fig. 2. It should be stressed that these complex responses are primitive behavioral operations for a reaction-diffusion medium and that they determine the character of primitive information processing operations that could be performed by the medium.

Image processing operations performed by active chemical media proved to be similar to the human visual capabilities and dependent on the state of the medium (Rambidi and Maximychev, 1995). There are two main sets of them.

The first of them can be defined as "description of the general features of an object". This set includes such primitive operations as concentration on the 


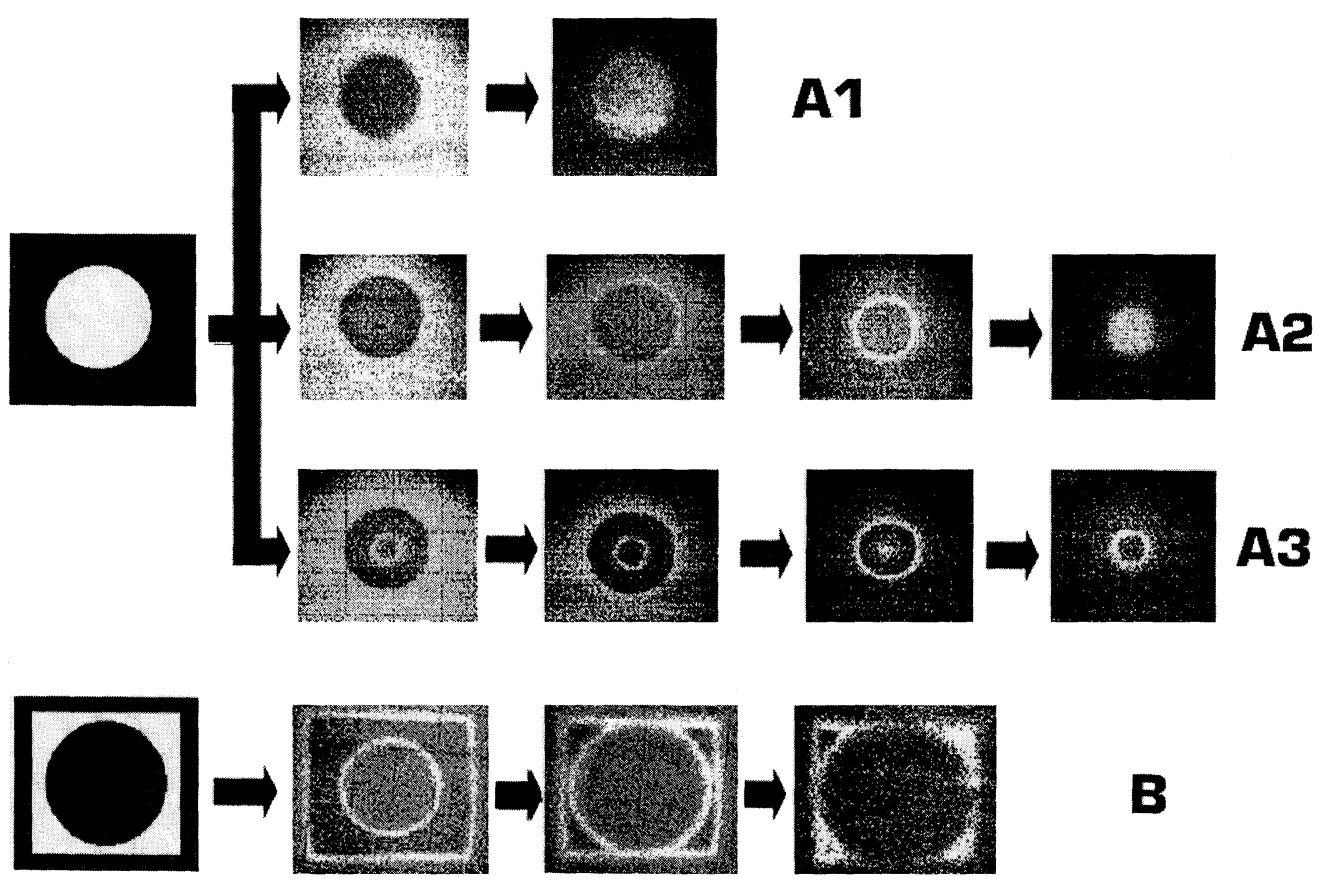

FIGURE 2 Temporal evolution of simple images (initial images are to the left) in thin layers of light-sensitive Belousov-Zhabotinsky type media depending on the state of the medium (A1-A3) and on the character of the input image (A-B).

general outline of an image (Fig. 3A), removing small immaterial features (Fig. 3B), "addition to the whole" operations, and, in particular, restoration of an image having defects (Fig. 3C).

The second set of image processing operations can be determined as "switching to the details of an image". It includes contour enhancement (Fig. 4A), segmentation, which is the division of an image into simple parts (Fig. 4B), image skeletonizing, italicizing small features of an image (Fig. 4C).

In the case of complex images having several levels of brightness primitive image processing operations proved to be complicated combinations of the basic responses of excitable media to the light excitation (Rambidi and Maximychev, 1997b).

It proved to be that in the process of the input image evolution all fragments having different brightness are consecutively enhanced at different stages of evolution.

Processing an image similar to pictures taken from satellites or reconnaissance planes are shown in Fig. 5. It could be seen that the most dark fragments are enhanced first in the process of evolution and after that the most light ones. Therefore the use of excitable media seems to be an attractive potential way for the processing of satellite or aerial information.

\section{MORE COMPLICATED PROBLEM: FINDING PATH IN A LABYRINTH}

During the last decades many proposals were made how to take effective solution for finding path in a labyrinth, one of the most known problems of high computational complexity inherent in information processing by biomolecular and biological entities. Proposals were particularly made to use technique attractive enough for solving this problem based on wave processes in reaction-diffusion media (see Rambidi and Yakovenchuk, 1999, and references to it). Trigger waves in reaction-diffusion systems spread simultaneously through all paths of the labyrinth in a highly parallel mode. This mode seems to be put into the basis of the computational 


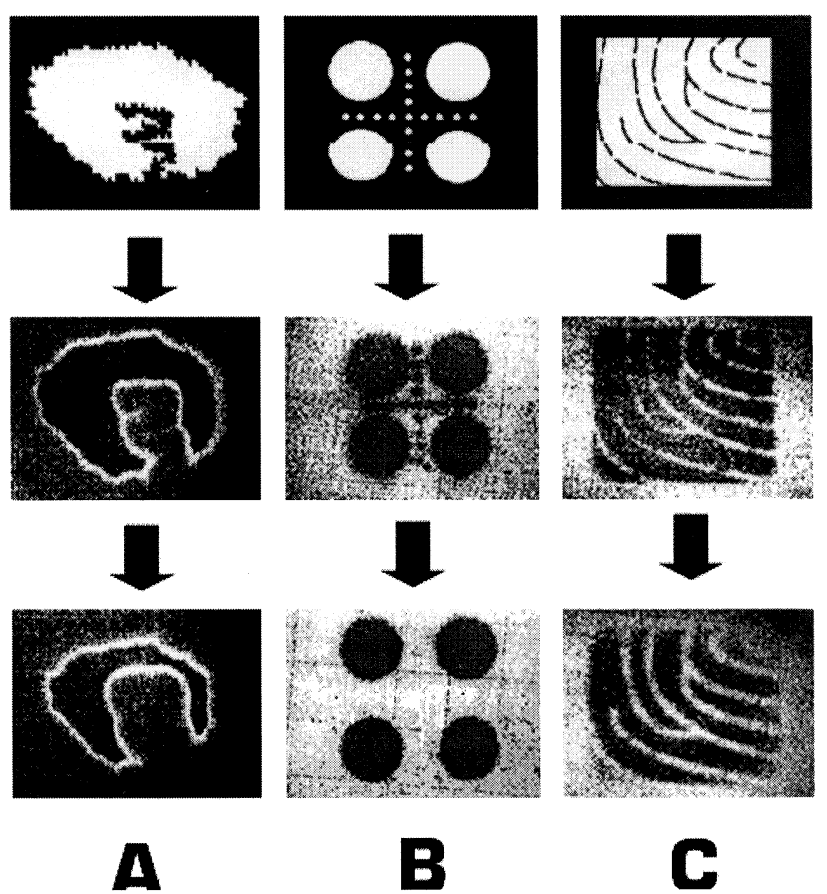

FIGURE 3 Some details of the temporal evolution of images in light-sensitive Belousov-Zhabotinsky type media and results of primitive image processing operations performed by the medium: (A) smoothing of immaterial features of the image; (B) removing of small features; (C) defect repair. Initial images are in the first row above, results are in the last row at the bottom.

technique for finding path in a labyrinth. Regretfully the velocity of these waves is extremely low that gave no way for practical implementation of this technique till now.

Effective "hardware" system was designed (Rambidi and Yakovenchuk, 1999) capable of finding path in a labyrinth using fast phase waves.

Three principal points were assumed as a basis for this design. They are the following:

(i) The information processing system based on reaction-diffusion mechanisms and capable of solving labyrinth problems should be of hybrid type. It should be a combination of reaction-diffusion medium with universal digital computer. This architecture enables to perform effectively the operations of high computational complexity (parallel spreading of a wave along all pathways of labyrinth, etc.) together with fast digital processing of intermediate data.
Let us make some remarks important for the understanding how to design effective computational procedure for finding paths in labyrinth.

Given computational character of the procedure the labyrinth should be stored in the memory as an image (in the simplest case, as black and white image, for instance, black picture of the labyrinth on the white background). Suppose that a starting point of a labyrinth only is defined and that a procedure is designed that would give the opportunity to record consecutive steps of the wave spreading in the computer memory. When the wave spreads along the path of the labyrinth black color of the labyrinth path changes to the color of the background. Some more or less sophisticated procedure could be developed that enables one to follow the wave front and to determine the point where the black labyrinth path would disappear. However it is impossible to distinguish between deadlocks and target points in this case. 

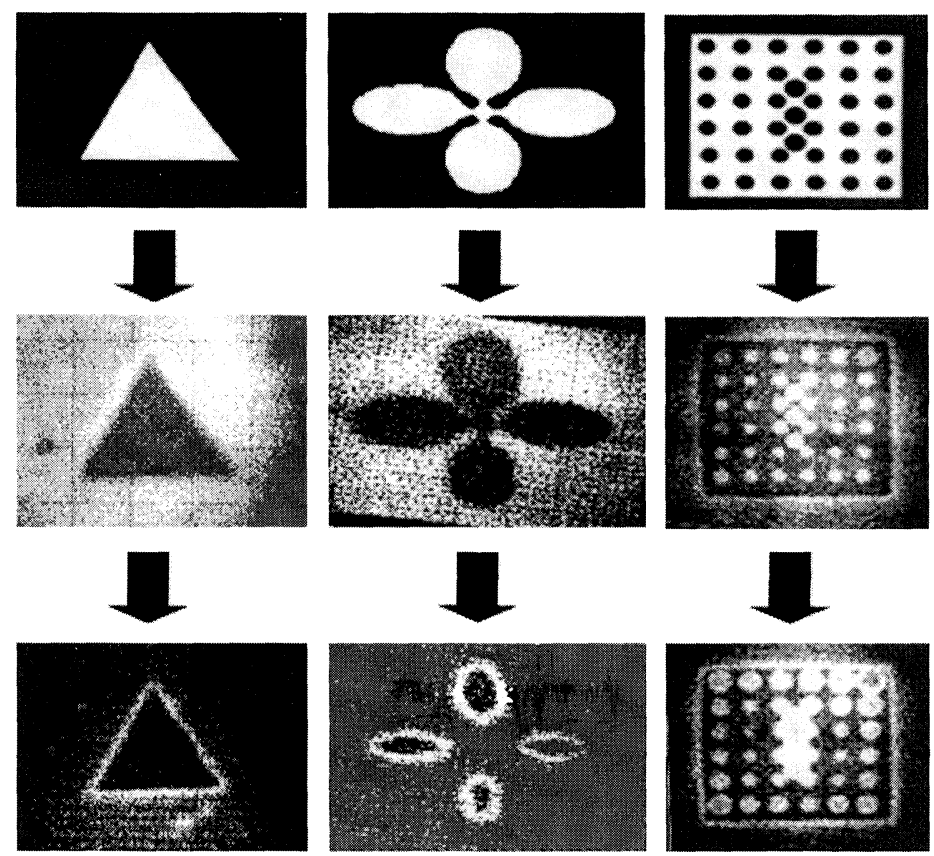

$\mathbf{A}$

$\mathbf{B}$

FIGURE 4 Some details of the temporal evolution of images in light-sensitive Belousov-Zhabotinsky type media and results of primitive image processing operations performed by the medium: (A) contour enhancement; (B) segmentation of an image; (C) enhancement of small features. Initial images are in the first row above, results are in the last row at the bottom.

Even if a human being moves trough a real labyrinth the difference between deadlocks and target points should be evident to leave the labyrinth.

Therefore, a starting point and target points should be defined to find a path in a labyrinth. More exactly some features should be included in the labyrinth image that mark target points and help to determine a moment when the wave would get the target point.

Supposing that the starting point and target points are known and also that trigger wave spreads along the labyrinth beginning from its starting point, the wave would eventually get the first target point nearest to the starting point and after that other target points would be successfully determined.

In this case, it is clear that the relative lengths of the paths only could be determined and not the paths themselves.

This consideration shows the main principal feature of the computational procedure for finding paths in a labyrinth based on wave processes inherent in nonlinear reaction-diffusion media.

The spreading of the wave through a labyrinth is a parallel operation of high computational complexity. A reaction-diffusion medium is able to perform effectively this operation and consecutive steps of it could be stored in a memory of digital computing system.

It would be shown below that computational digital procedure of low computational complexity could be offered capable of finding paths in a labyrinth and based on the set of data stored in the computer memory that describe spreading wave through the labyrinth.

(ii) The most important problem is how to store a labyrinth of arbitrary structure in the reactiondiffusion medium for its further processing.

Light sensitive Belousov-Zhabotinsky type reagent seems to be one of the most suitable media 


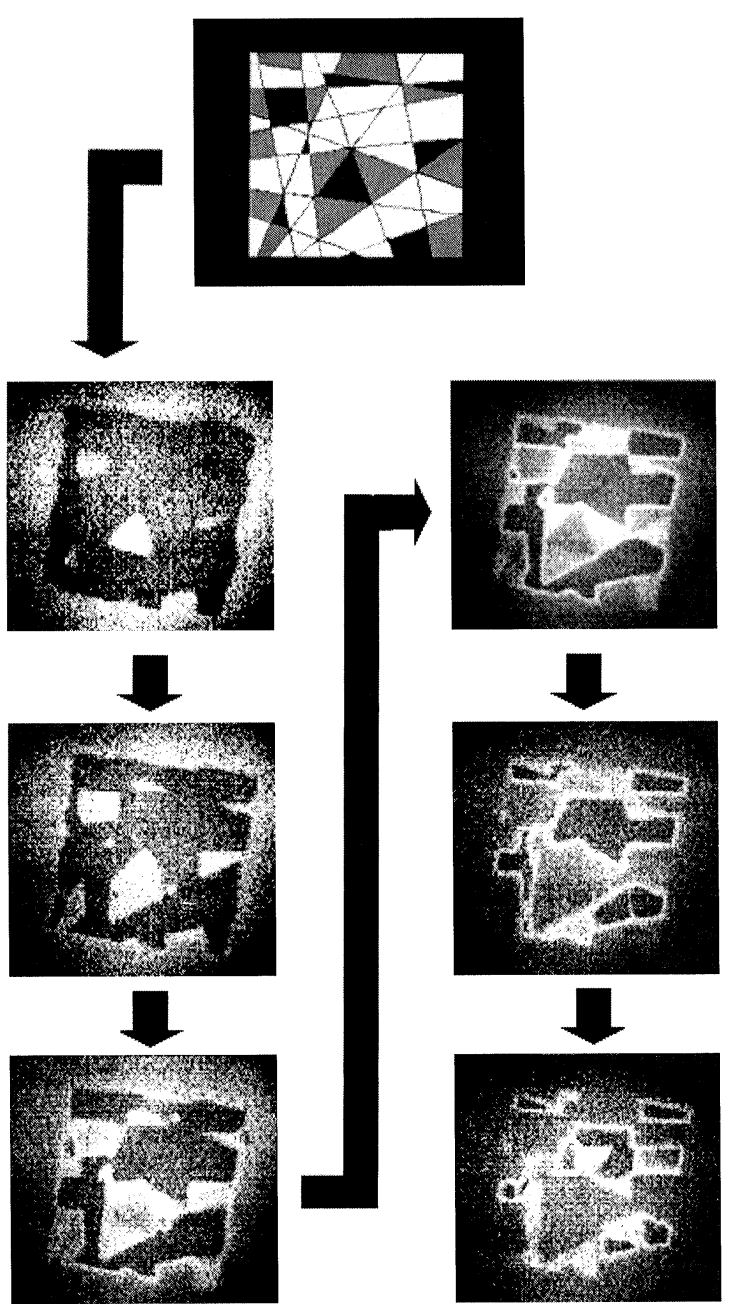

FIGURE 5 Evolution of artificial aerial picture in a light-sensitive Belousov-Zhabotinsky type medium.

for solving labyrinth problems because the key feature of light-sensitive excitable media is that they store input information for a rather long period of time (see Rambidi, 1997, and references to it). The initial labyrinth image and steps of its further transformation in the medium (that is spreading wave evolution) could be detected by a video camera and stored in a digital computer memory. After that finding the shortest paths in a labyrinth could be reduced to image processing operations.

(iii) The main and decisive point of the problem under consideration is how to organize a wave process capable to spread in a parallel mode trough all possible paths of a labyrinth.

Two kinds of traveling processes inherent in interaction-diffusion systems are known. The first of these represents propagation of trigger waves due to the interaction of chemical reaction and diffusion of reaction components. The velocity of the trigger waves is very low $(\sim 0.05 \mathrm{~mm} / \mathrm{s})$. The second of these, so called phase waves, propagate independently of diffusion along a phase gradient in an oscillatory medium. The phase waves are fast, but difficult to control.

The remarkable property of the Ru-catalyzed Belousov-Zhabotinsky type media is a possibility to control phase processes proceeded in the medium by the light radiation (Rambidi et al., 1994).

Fast light-induced phase wave processes were used in this work that spread through the labyrinth in several seconds instead of hours typically for trigger waves inherent in reaction-diffusion media.

These fundamentals along with additional procedure of testing for labyrinth fragments connectedness gave the opportunity to solve labyrinth problems. Let us consider this technique for the simplest case of linear tree-like labyrinth (Fig. 6).

The wave spreading in the labyrinth starting from the point of input to the output is shown in Fig. 6 . Since this process is taking place for about $3-5 \mathrm{~s}$ it is easy to record consecutive steps of this spreading. Some of them are shown in Fig. 6.

Taking a set of consecutive images of the wave spreading the basic problem arises: how to use this data for finding a path from the input to output.

In the process of the wave spreading when the wave goes over a branching point the labyrinth is divided into two (or more) fragments. One of these is connected with the output, but the others are not. It is easy to find the fragment connected with the output initiated backward wave from the point of output in reaction-diffusion medium. As a result this fragment changes its color and the color of the nonconnected fragment only remains unchanged.

More effective solution is to change this auxiliary reaction-diffusion process by numerical processing the image of the labyrinth at this step of its evolution 


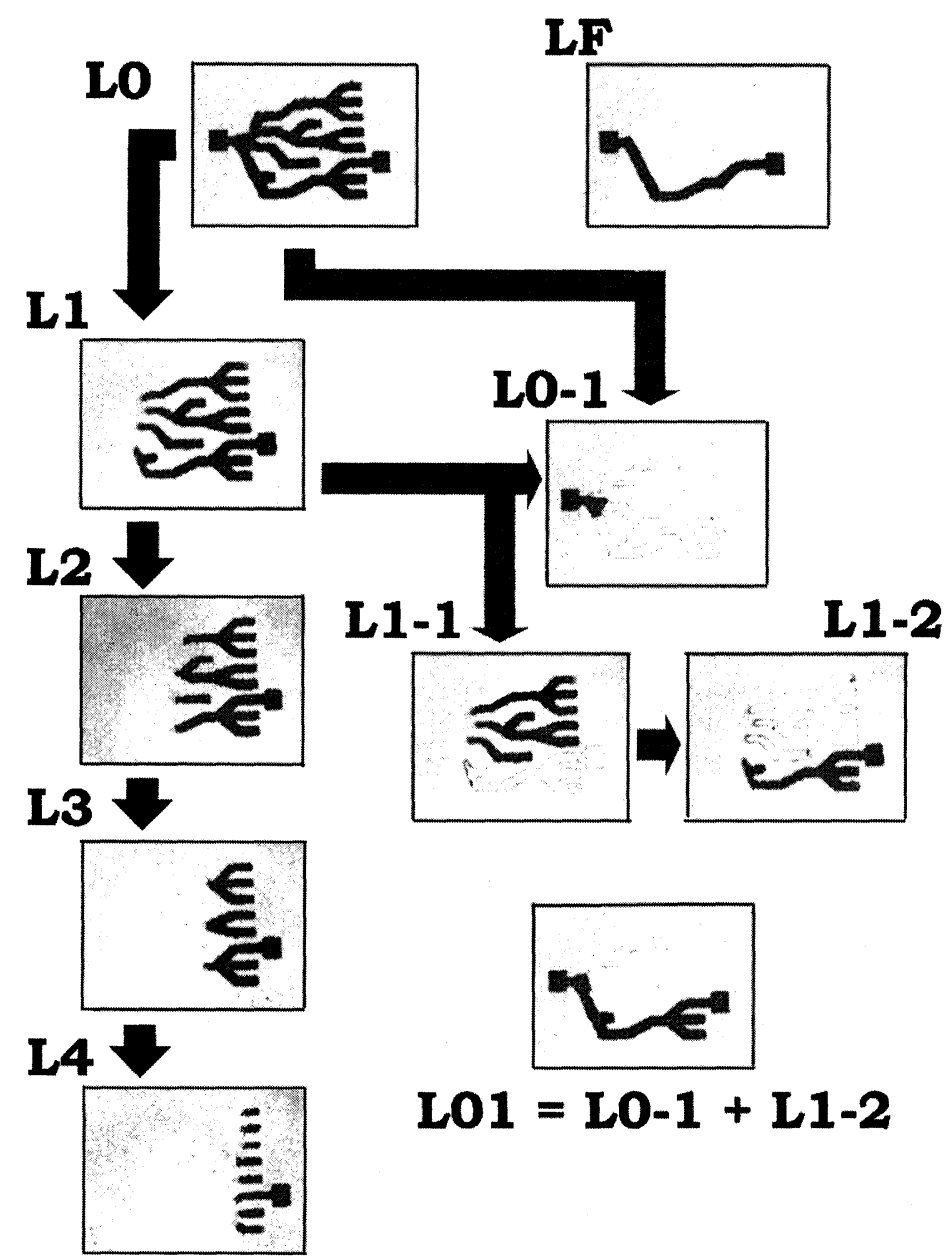

FIGURE 6 Finding shortest path in a simple tree-type labyrinth. L0 is an initial image of the labyrinth in the Belousov-Zhabotinsky type medium, L1-L4: some consecutive steps of its evolution in the process of the wave spreading, LF: the image of the shortest path in the labyrinth, $\mathrm{L} 0-1$ is the pathway which the wave has passed during the first step of its spreading, $\mathrm{L} 1-1$ is the result of Paint Bucket operations for L1 image, L1-2 presents result of subtraction of non-connected with target point parts from the L1 image.

stored in the memory of the computer, namely to use Paint Bucket operation initiated at the point of output from the labyrinth. This operation changes the color of this fragment to the color of a background. Subtracting the image obtained after Paint Bucket operation from the initial labyrinth image enables to remove fragment not connected with the output.

Successive repetition of this procedure at each branching point gives the opportunity to exclude all fragments coming to deadlocks (and to other outputs in general case) and to determine the path from the input to the output.

\section{NUMERICAL MODELING: FROM THE GENERATION OF BUTTON TEXTURES UP TO IMAGE PROCESSING}

The variety of reaction-diffusion simulations performed during the last several decades is rather broad.

The first fundamental work in this field was the paper by great Alan Turing titled "The chemical basis of morphogenesis" (Turing, 1952). It was the simulations of the differentiation processes based on nonlinear kinetic equations describing a set of coupled 
chemical reactions. It was also one of the first examples shown that nonlinear dynamic mechanisms could be in the basis of high behavioral complexity of the biological system.

This biological line of reaction-diffusion simulations have been continued till now including the broad scope of problems beginning from models of biological pattern formation such as giraffe or zebra skin patterns up to applications to population dynamics (see, for instance, Turk, 1991, and references to it).

Similar technique was used for purely practical purposes such as computer graphics (see Witkin and Kass, 1991). It gave the opportunity to generate different reaction-diffusion textures for picturesque buttons, specific art painting and so on. It should be mentioned that high behavioral complexity of reaction-diffusion media revealing in textures seems to be virtually in the basis of the human sense of beauty.

Very important were repeated approaches to simulate regimes inherent in Belouso-Zhabotinsky and other reaction-diffusion media. They enabled to calculate different reaction-diffusion patterns including evolution of breathing spots, spiral and labyrinthine patterns (for instance, Hagberg and Meron, 1994; Haim et al., 1996).

Three-dimensional reaction-diffusion systems represent fantastic pictures of complex behavior (Winfree, 1992). Basic results in this attractive and promising field were obtained by numerical simulation because of big difficulties in obtaining reliable experimental information. Apart from general importance of the field these investigations are indispensable for understanding features of dynamic mechanisms inherent in cardiac muscle and nerve network activity.

The simulation of image processing capabilities of reaction-diffusion systems is another remarkable direction of investigations (Price et al., 1990; Bellustin et al., 1991; Kuznetsov et al., 1998).

Yakhno with coworkers (Bellustin et al., 1991; Kuznetsov et al., 1998) used Eq. (2) that describes the media having short-range nonlocal interactions. They found out that simulation is capable to describe a number of image processing operations (see, for instance, Fig. 7). They embrace:

- contour enhancement;

- transformation of halftone images into high contrast ones;

- image skeletonizing;

- extraction of lines having a given direction;

- calculation of invariant features.

Some of these results were obtained also a little earlier by Price et al. (1990).

Let us mention that these examples show some practical advantage of numerical simulations of reaction-diffusion system activity in comparison with experimental studies.

Contrary to experimental investigations numerical simulations give the opportunity to use unreal medium characteristics (for instance, anisotropic coupling functions) and to model regimes that can not be reproduced in experiment. As a result the system proves to be able to perform more sophisticated information processing operations such as enhancement of sharp corners of an image or lines turned to predetermined directions. In other words the following sequence of steps is seen: complication of medium dynamic characteristics-increasing behavioral complexity-performing more complicated information processing operations.

These simulations are in a good correlation with experimental results conforming at the theoretical level the close correlation between behavioral complexity of the system and its ability to solve problems of high computational complexity.

Cellular automata technique was an important part of the numerical simulations of reaction-diffusion media.

Between different realization of this technique two directions of the investigations should be mentioned.

Tyson and his coworkers succeeded to simulate complicated modes of Belousov-Zhabotinsky type media (see, for instance, Weimar et al., 1992, and references to it).

Adamatzky used cellular automata calculations to analyze important characteristics of reaction-diffusion media and potentialities of their practical use (Adamatzky, 1998, and references to it). 

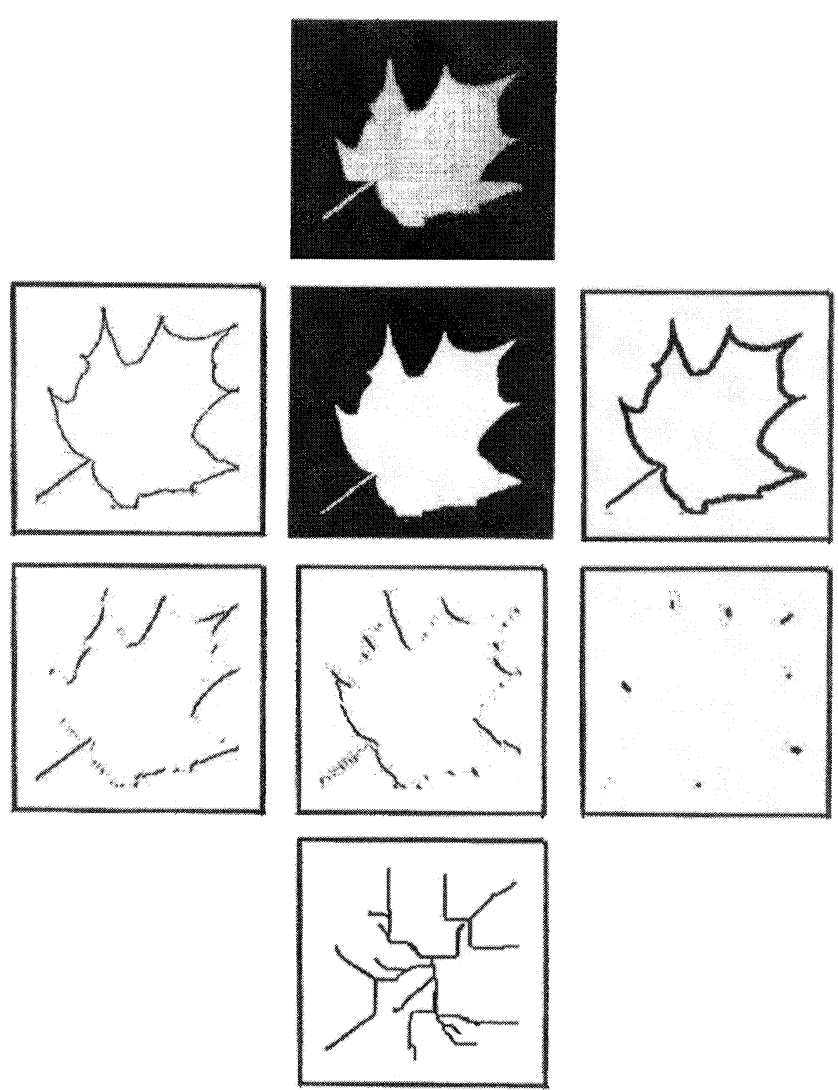

FIGURE 7 Numerical simulation of image processing based on reaction-diffusion equations. Initial half-tone image is shown in upper part of the picture. Results of simulation different due to divers choice of coupling functions and shifts are: enhancement of thin and thick contour, contrast enhancement, enhancement of lines having different slope and corners of the image, skeleton of the image.

\section{FUTURE IMPLICATIONS-TOWARDS A BIOMOLECULAR COMPUTER}

An attempt was made above to show that high behavioral complexity of the biomolecular systems could be correlated with the ability of the system to solve problems of high computational complexity. The natural further evolution of this assumption is to suppose that molecular dynamic systems having more complicated behavior than discussed above would be capable of solving much more complicated problems.

Separate considerations show that potential implementations of molecular dynamic systems are far from exhausted.

In the late 1980s, Conrad (1989) discussed in detail the analogy and disanalogy of information features of the brain and digital von Neumann computer. In the background of this analysis lies the tradeoff principle formulated by Conrad (1985):

"A system cannot at the same time be effectively programmable at the level of structure, amendable to evolution by variation and selection, and comutationally efficient."

Proceeding from the tradeoff principle Conrad paid attention just to those differences between the information features of the brain and von Neumann computers that are of fundamental significance (see Fig. 8). He concluded that according to these differences von Neumann computers could not pass the Turing test (Turing, 1950), i.e. that the intellect of the brain differs appreciably from the possible degree of von Neumann computer intellect. 
Conrad (1989) stressed that information processing devices based on principles of information processing at the molecular level should possess information features much more similar to the information features of the brain. Therefore, it seems reasonable to consider information features of reaction-diffusion systems that can be now designed utilizing contemporary laboratory technology (Rambidi, 1994).

Let us choose such devices as the reactiondiffusion system formed on the basis BelousovZhabotinsky media. Let us consider the basic information features of this device.

First, they include a high degree of organization inherent in molecular media of this type. Moreover, these media display gradualism, i.e. small changes of active medium composition, within define limits, lead only to small quantitative, not qualitative changes of system dynamics.

It is essential that media of Belousov-Zhzabotinsky type have also other characteristics necessary, according to Ashby (1960), for displaying adaptive behavior. Among these is the nature of interaction of the system with the environment, feedback organization, etc. Therefore it is possible to conclude that it could really be possible to create a device, capable of learning, having multilevel architecture and a high degree of behavioral complexity.

It is easy to see that the lack of structural programmability, a high degree of parallelism, mixed continuous and discrete dynamics, and highly developed coupling between elements of the medium are typical for reaction-diffusion means (see Fig. 8).

Even simple reaction-diffusion systems display a vertical flow of transmission and processing information. Even in a simple system the following can be determined.

- the level of macro-micro type transformation of information can be determined: (particularly, photochemical transformation of a light twodimensional picture into pseudo-two-dimensional distribution of reaction components);

- dynamics at the molecular level implementing a definite information process;

\begin{tabular}{|c|c|c|}
\hline & & \\
\hline & & \\
\hline & & \\
\hline $\begin{array}{l}\text { PAOGRAMMED } \\
\text { FROM OUTSIDE }\end{array}$ & $\begin{array}{l}\text { SELF- } \\
\text { ORGANIZING }\end{array}$ & $\begin{array}{l}\text { SELF- } \\
\text { ORGAMIZINO }\end{array}$ \\
\hline STRUCTURALLY & STRUCTURALLY & STRUCTURALLY \\
\hline PFOQRAMMABLE & NON- & NON- \\
\hline & PROQRAMMABLE & PROGRAMMABLE \\
\hline SEQUENTIAL & HIGHLY & MASSIVELY \\
\hline USE OF & PARALLEL & PARALLEL \\
\hline BESOURCES & & \\
\hline DISCRETE & CONTINUOUS & DISCRETE AND \\
\hline DYNAMICS & AND DI8CRETE & CONTINUOUS \\
\hline & DYNAMICS & DYNAMICS \\
\hline HIGHLY & HIOHLY & HIGHLY \\
\hline CONSTRAINED & INTERACTIVE & INTERACTIVE \\
\hline HORIZONTAL & VERTICAL & VERTICAL \\
\hline FLOW OF & INFORMATION & INFORMATION \\
\hline INFORMATION & FLOW & FLOW \\
\hline
\end{tabular}

FIGURE 8 Brain []-machine []-reaction-diffusion processor [?] analogies-disanalogies.

- the level of micro-macro information transformation, i.e. physico-chemical reading information.

Unlike the digital von Neumann computer, the reaction-diffusion device is not a rigid structurally predetermined system. Its dynamics depends on the composition and controls stimuli variations. It enables implementation of rather effective control of the dynamics.

In general, the detailed comparison of the information features of the brain, the digital von Neumann computer and reaction-diffusion media leads to the conclusion that there is a sufficiently greater analogy between features of reaction-diffusion media and the brain, which is the system that solves problems of high computational complexity in a natural way.

From considerations discussed above it seems to follow that it is the complicated nonlinear dynamics 
that determine the information features inherent in reaction-diffusion biomolecular devices. At the same time the surprising resemblance of features of the brain and simple enough reaction-diffusion systems that determine the general principles of information processing (self-organization, high parallelism, vertical flow of information, etc.) is striking.

The brain is immeasurably richer in its functions than the specialized reaction-diffusion device. The completeness of solving intellectual problems by brain exceeds all that can be reached nowadays by any "man-made" device. Nevertheless, the analogy of information processing features seems to be the evidence that there exist a set of logical operations inherent in reaction-diffusion systems that are optimal and whose mechanisms are close to the analogous mechanisms of the brain.

There are two general ways to realize these advanced capabilities.

First of these is to look for sophisticated dynamic mechanisms that would be capable of increasing the behavioral complexity of the system and as a consequence the computational complexity of problems solving by the system.

Experimental data and theoretical considerations shows that this approach seems to open new potentialities for practical implementation of complex information processing operations (see for instance, Michailov, 1993).

The second one represents the designing of complicated architectures, particularly multilevel biomolecular information processing systems.

There were a number of experimental investigations and theoretical estimations that open the practical approaches to designing these systems (see Epstein, 1990; Laplante and Erneux, 1992; Hjelmfelt et al., 1993; Hjelmfelt and Ross, 1994; Laplante et al., 1995; Dechert et al., 1996).

Experimental technique for finding path in a labyrinth is one of the examples of this approach implementation. The main and decisive feature of this system is its two-level character, that is dividing the system into two parts representing the catalyst immobilized on the solid support and all other components of the chemical system in liquid phase.
This short and superficial enough survey of reaction-diffusion media potentialities shows that this attractive and promising field would give important practical results in not so distant future. And that complexity background is one of keystones for the progress of the field.

\section{References}

Adamatzky, A. (1998) "Dynamical universal computations in excitable lattices", in: Morgensten, M. (Ed.), Proceedings of Second International Colloquium Universal Machines and Computations, Vol. 2, pp. 194-214.

Ashby, W.R. (1960) Design for a brain (Chapman \& Hall, London),

Beer, S. (1970) "Managing modern complexity", Futures 2, 245-257.

Bellustin, N.S., Kuznetsov, S.O., Nuidel, I.V. and Yakhno, V.G. (1991) "Neural networks with close nonlocal coupling for analyzing composite images", Neurocomputing 3, 231-246.

Casti, J. (1979) Connectivity, Complexity, and Catastrophe in Large-Scale Systems (Wiley, New York), p 44.

Conrad, M. (1985) "On design principles for a molecular computing", Commun. ACM 28, 464-480.

Conrad, M. (1989) "The brain-machine disanalogy", BioSystems 22, 197-213.

Davydov, A.S. (1984) Solitons in Molecular Systems (Naukova Dumka, Kiev), in Russian.

Dechert, G., Zeyer, K.P., Lebender, D. and Schneider, F.W. (1996) "Recognition of phase patterns in a chemical reactor network", J. Phys. Chem. 100, 19043-19048.

Epstein, I.R. (1990) "Experimental and theoretical studies of coupled chemical oscillators", React. Kinet. Catal. Lett. 42, 241-252.

Kapral, R., Showalter, K. (1985) In: Oscillations and traveling waves in chemical systems (Wiley/Interscience, New York).

Goldbetter, A. (1997) Biochemical Oscillations and Cellular Rhythms (Cambridge University Press, Cambridge).

Grossberg, S. (1976) "On the development of feature detectors in the visual cortex with applications in learning and reactiondiffusion systems", Biol. Cybernetics 21, 145-159.

Grossberg, S. (1988) "Nonlinear neural networks: principles, mechanics and architecture", Neural Networks 1, 59-64.

Hagberg, A. and Meron, E. (1994) "From labyrinthine patterns to spiral turbulence", Chaos 4, 477.

Haim, D., Li, G., Quyang, Q., McCormick, W.D., Swinney, H.L., Hagberg, A. and Meron, E. (1996) "Breathing spots in reactiondiffusion systems", Phys. Rev. Lett. 77, 190.

Hjelmfelt, A. and Ross, J. (1994) "Pattern recognition, chaos, and multiplicity in neural networks of excitable systems", Proc. Natl. Acad. Sci. USA 91, 63-67.

Hjelmfelt, A., Schneider, F.W. and Ross, J. (1993) "Pattern recognition in coupled chemical kinetic systems", Science $\mathbf{2 6 0}$, 335-337.

Kapral, R., Showalter, K. (1995) In: Chemical Waves and Patterns (Kluwer Academic Publishers, Dordrecht).

Kuznetsov, S.O., Nuidel, I.V., Panfilov, A.I. and Yakhno, V.G. (1998) "Image preprocessing by neuron-like algorithms", SPIE Proc. 3402, 479-485. 
Laplante, J.P. and Erneux, T. (1992) "Propagation failure and multiple steady states in an array of diffusion coupled flow reactors", Physica A 188, 89-98.

Laplante, J.P., Pemberton, M., Hjelmfelt, A. and Ross, J. (1995) "Experiments on pattern recognition by chemical kinetics", J. Phys. Chem. 99, 10063-10065.

Lee James, S.I., Nguyen Dziem, D. and Lin, C. (1989) “Adaptive moving object tracking integrating neural networks and intelligent processing", SPIE Sem. Proc. 1002, 316-323.

Masterov, A.V., Rabinovich, M.I., Tolkov, V.N. and Yakhno, V.G. (1988) "Studies of regimes of autowaves and autostructures interaction in neural net like media", In: Yakhno, V.G., ed, Collective Dynamics of Excitations and Structure Formation (Institute of Applied Physics of the USSR Academy of Sciences, Gorky), in Russian, pp 89-104.

McCulloch, J. and Pitts, W. (1943) "A logical calculus of the ideas immanent in nervous activity", Bull. Math. Biophys. 5, 115-133.

Michailov, A.S. (1993) "Collective dynamics in communicated populations", In: Haken, H. and Michailov, A.S., eds, Interdisciplinary Approaches to Nonlinear Complex Systems (Springer, New York), pp 89-108.

Ming, L. and Vitanyi, P. (1993) An Introduction in Kolmogorov Complexity and it's Applications (Springer, New York).

Nicolis, J.S. (1986) Dynamics of Hierarchical Systems. An Evolutionary Approach (Springer, Berlin).

Niels, R. and Hepner, G. (1990) "Application of an artificial neural networks to landcover classification of thematic mapper imagery", Comput. Geosci. 16, 873-880.

Price, C.B., Wambacq, P. and Oosterlink, A. (1990) "Image enhancement and analysis with reaction-diffusion paradigm", IEE Proc. 137, 136-145.

Prigogine, I. (1980) From Being to Becoming: Time and Complexity in the Physical Sciences (Freeman, San Francisco).

Rambidi, N.G. (1994) "Biomolecular computing: from the brainmachine disanalogy to the brain-machine analogy", BioSystems 33, 45-54.

Rambidi, N.G. (1997) "Biomolecular computer: roots and promises", BioSystems 44, 1-15.

Rambidi, N.G. and Maximychev, A.V. (1995) "Molecular image processing devices based on chemical reaction systems 3: some operational characteristics of excitable light-sensitive media used for image processing", Adv. Mater. Opt. Electron. 5, 223-231.

Rambidi, N.G. and Maximychev, A.V. (1997a) "Molecular image processing devices based on chemical reaction systems 6: processing half-tone images and neural network architecture of excitable media", Adv. Mater. Opt. Electron. 7, 171-182.

Rambidi, N.G. and Maximychev, A.V. (1997b) "Molecular image processing devices based on chemical reaction systems 5: processing images with several levels of brightness and some application potentialities", Adv. Mater. Opt. Electron. 7, 161-170.

Rambidi, N.G. and Yakovenchuk, D. (1999) "Informationprocessing capabilities of chemical reaction-diffusion systems 2: finding paths in a labyrinth based on reaction-diffusion media", Adv. Mater. Opt. Electron. 9, 27-34.

Rambidi, N.G., Maximychev, A.V. and Usatov, A.V. (1994) "Molecular image processing devices based on chemical reaction systems 1: general principles for implementation", Adv. Mater. Opt. Electron. 4, 179-190.

Rambidi, N.G., Kuular, T.O.-O. and Machaeva, E.E. (1998) "Information-processing capabilities of chemical reactiondiffusion systems 1 . Belousov-Zhabotinsky media in hydrogel matrices and on solid supports", Adv. Mater. Opt. Electron. 8, $163-171$.

Trouder, T. and Merril, W. (1990) "A real-time neural-net estimator of fatigue life", Int. Joint Conf. Neural Networks 2, 59-64.

Turing, A.M. (1950) "Computing machinery and intelligence", Mind 59, 433-460.

Turing, A.M. (1952) "The chemical bases of morphogenesis", Philos. Trans. R. Soc. Lond. B327, 37-72.

Turk, G. (1991) "Generating textures on arbitrary surfaces using reaction-diffusion", Comput. Graphics 25, 289-298.

Vasilev, Y.A., Romanovsky, Yu.M., Chernavskii, D.S. and Yakhno, V.G. (1987) Autowave Processes in Kinetic Systems (VEB Deutscher Verlag der Wissenschaften, Berlin).

Weimar, J.R., Tyson, J.J. and Watson, L.T. (1992) "Diffusion and wave propagation in cellular automaton model of excitable media", Physica D 55, 309-327.

Winfree, A.T. (1992) "The geometry of excitability", In: Nadel, L. and Stein, D., eds, Lectures in Complex Systems (Addison Wesley, Reading, MA), p 1993.

Winfree, A.T. (1994) "Electrical turbulence in three-dimensional heart muscle", Science 266, 1003-1006.

Witkin, A. and Kass, M. (1991) "Reaction-diffusion textures", Comput. Graphics 25, 299-308.

Yudin, D.B. and Yudin, A.D. (1985) The Number and the Thought (Znanie, Moscow), in Russian, pp 26-35.

Zhabotinsky, A.M. (1964) "Periodic processes of malonic acid oxidation in a liquid phase", Biofizika 9, 306. 


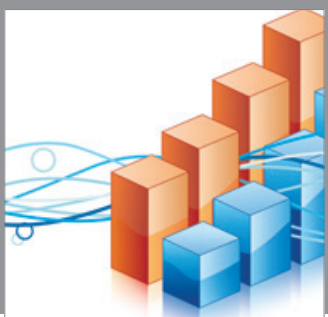

Advances in

Operations Research

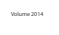

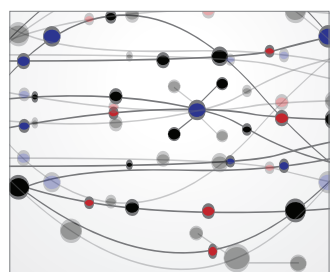

\section{The Scientific} World Journal
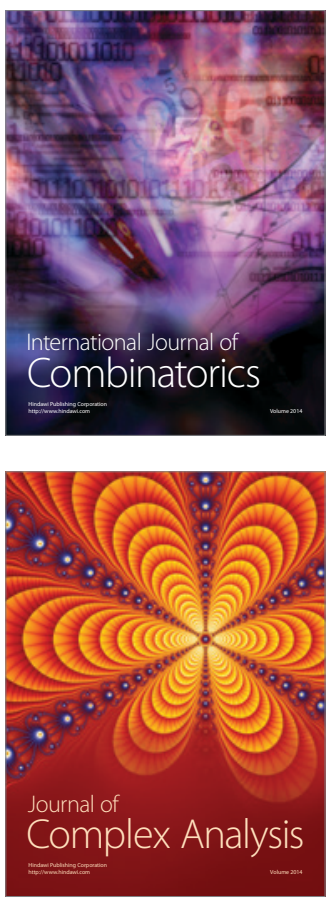

International Journal of

Mathematics and

Mathematical

Sciences
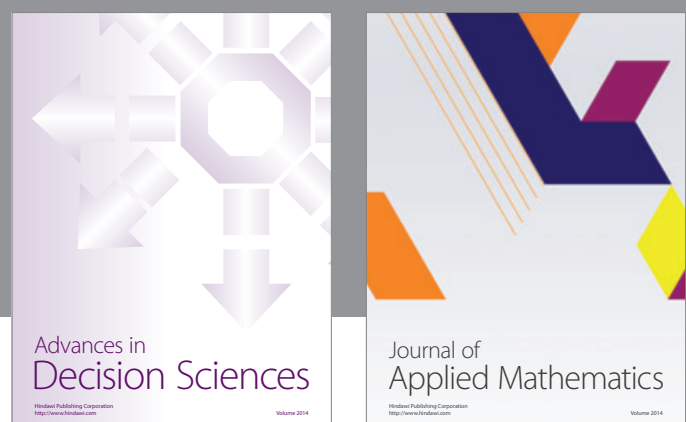

Journal of

Applied Mathematics
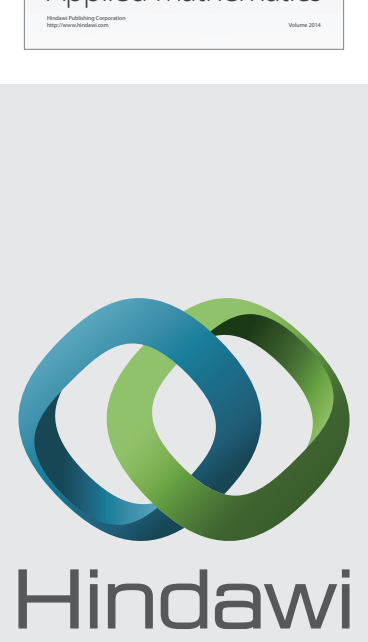

Submit your manuscripts at http://www.hindawi.com
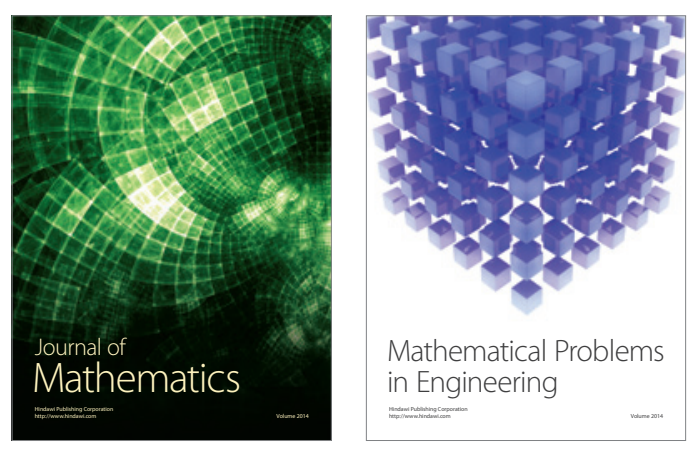

Mathematical Problems in Engineering
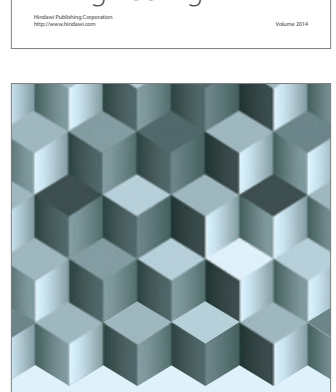

Journal of

Function Spaces
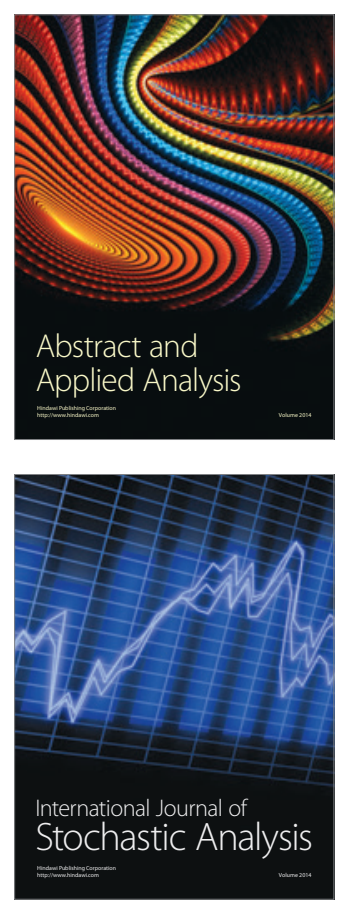

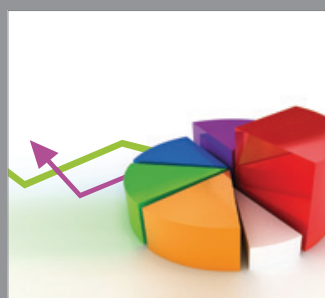

ournal of

Probability and Statistics

Promensencen
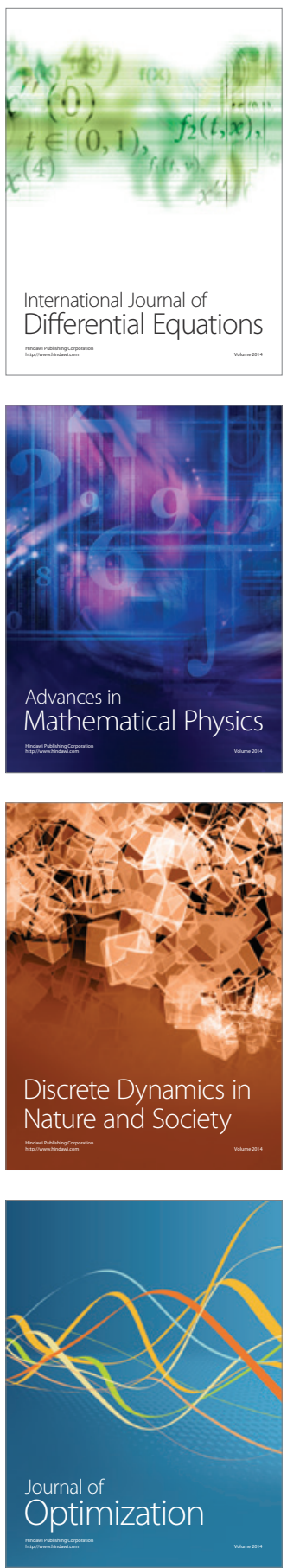